\title{
Review Article \\ Clinical Practice Guidelines in Psychiatry: More Confusion Than Clarity? A Critical Review and Recommendation of a Unified Guideline
}

\author{
Sahoo Saddichha ${ }^{1}$ and Santosh K. Chaturvedi ${ }^{2}$ \\ ${ }^{1}$ NWMH, Melbourne Health, 4a Devonshire Road, Sunshine, VIC 3020, Australia \\ ${ }^{2}$ Department of Psychiatry, National Institute of Mental Health \& Neurosciences (NIMHANS), Bangalore 560029, India \\ Correspondence should be addressed to Sahoo Saddichha; saddichha@gmail.com
}

Received 9 January 2014; Accepted 12 March 2014; Published 31 March 2014

Academic Editors: M. Bernardo and A. Michael

Copyright (C) 2014 S. Saddichha and S. K. Chaturvedi. This is an open access article distributed under the Creative Commons Attribution License, which permits unrestricted use, distribution, and reproduction in any medium, provided the original work is properly cited.

\begin{abstract}
The discipline of psychiatry has a plethora of guidelines, designed to serve the needs of the clinician. Yet, even a cursory glance is enough to discern the differences between the various guidelines. This paper reviews the current standard guidelines being followed across the world and proposes a unified guideline on the backbone of current evidence and practice being followed. The algorithm for pharmacological and psychosocial treatment for bipolar disorder, major depressive disorder, and schizophrenia is formulated after cross-comparison across four different guidelines and recent meta-analytical evidence. For every disorder, guidelines have different suggestions. Hence, based on the current status of evidence, algorithms have been combined to form a unified guideline for management. Clinical practice guidelines form the basis of standard clinical practice for all disciplines of medicine, including psychiatry. Yet, they are often not read or followed because of poor quality or because of barriers to implementation due to either lack of agreement or ambiguity. A unified guideline can go a long way in helping clear some of the confusion that has crept in due to the use of different guidelines across the world.
\end{abstract}

\section{Introduction}

Standard clinical practice is considered the hallmark of every clinician, which is usually dictated by adhering to certain clinical guidelines [1]. A good guideline should be able to (a) identify the key decisions (e.g., diagnosis, assessment strategy, and treatment choice), (b) review the relevant, valid evidence on the benefits, risks, and costs of alternative decisions, and (c) present recommendations in a concise, updated format $[1,2]$. Guidelines should use best evidence available as well as having the flexibility of being regularly updated, without leaving any scope for ambiguity. They have, therefore, the best potential for ensuring that rigorous clinical standards are maintained and "best practice" is followed by clinicians [2]. Yet, they are often not read or followed because of poor quality $[3,4]$ or because of barriers to implementation due to either lack of agreement or ambiguity [5]. This issue becomes even more important for trainee residents who are on their way to become consultants as they are required not only to adhere to guidelines while practicing, but also to be fully aware of them during their clinical evaluation.

Psychiatry, as a discipline, is relatively new in the field of evidence based medicine; yet, there exist several clinical guidelines designed to provide guidance of good clinical practice. Some of the most well-known and widely accepted guidelines are the APA guidelines by the American Psychiatric Association [6], the Canadian treatment guidelines by the Canadian Psychiatric Association $[7,8]$ and the CANMAT (Canadian Network for Mood and Anxiety Treatments) $[9,10]$, the NICE guidelines by National Institute for Clinical Excellence [11], and the Maudsley guidelines [12]. As should be expected, all the above mentioned guidelines need to be uniform and precise. Yet, as this critique shows, there are several differences when these guidelines are compared. This paper, therefore, attempts to compare the treatment recommendations by the above mentioned guidelines for schizophrenia, major depression, and bipolar disorder, which are often considered as major mental disorders, and evaluates 
the differences between them. It also, ultimately, suggests a unified guideline putting together the common recommendations and available evidence from all the above.

\section{Methodology}

A Medline search on practice guidelines using terms "schizophrenia," "depressive disorder," "bipolar disorder," "management," "review," and combinations thereof helped us retrieve relevant literature. In addition, salient references from obtained articles were followed upon. Standard textbooks of psychiatry (Kaplan \& Sadock's Textbook of Psychiatry, Oxford Textbook of Psychiatry and Tasman \& Kay's Textbook of Psychiatry) were also screened using the same keywords. The most common guidelines, namely, the CPA, the APA, the CANMAT, NICE, and the Maudsley guidelines, were also reviewed. Additionally, searches were performed in PsycINFO, Medline, Cochrane, and Embase databases for reviewing guidelines for management until June 2011. All literature compiled was then assessed by the authors separately and an algorithm was proposed for each psychiatric disorder, taking into consideration the various guidelines proposed for the management of the specific disorder.

\section{Results}

\subsection{Management of Bipolar Disorder (Table 1)}

3.1.1. Acute Management of Manic Episode. Several differences are apparent in the acute management of a manic episode among the various guidelines. While both the APA and Maudsley prefer mood stabilizers like Lithium or Valproate as first line management, NICE and the Canadian guidelines prefer the use of atypical antipsychotics like Olanzapine (Olanz/OLZ) or Risperidone (Risp). Recent reviews favor the use of either Lithium $(\mathrm{Li})$ or Sodium Valproate (Valp) with/without antipsychotic (Level I evidence) in the management of the acute episode $[13,14]$. Since NICE also mentions the utility of both Li and Valp, a unified recommendation is to use Li or Valp as first line and consider Olanz or Risp next.

3.1.2. Acute Management of Mixed Episode. There are no differences of opinion here with all guidelines and hence the unified guideline recommends the use of Valp as a first line management followed by Olanz or Quetiapine (Quet) (Level II evidence $[13,14])$.

3.1.3. Acute Management of Depressive Episode. There are minor differences of opinion here, with Li being considered as first line by all the guidelines. However, while the use of Lamotrigine is favored by APA, Maudsley, and the Canadian guidelines, NICE discourages it and prefers a combination of Specific Serotonin Reuptake Inhibitors (SSRIs) with either Li or Valp. The combination of Olanz and Fluoxetine (FXT) has been recommended by almost all guidelines except by NICE, while Quetiapine is universally accepted. Yet, the evidence in favor of these is that of Level II $[14,15]$. Therefore the unified guideline recommends the use of $\mathrm{Li}$, either stand-alone or in combination with an SSRI, followed by either Quetiapine or Lamotrigine or the use of Olanz-FXT combination [16].

3.1.4. Acute Management of Rapid Cycling. Only the APA and NICE guidelines comment on this issue with both recommending either $\mathrm{Li}$ or Valp or a combination of the two, with Level II evidence available for the same [14]. Additionally, Olanz/Lamotrigine or Quetiapine (Levels II-III evidence $[14,17])$ may also be used.

3.1.5. Prophylaxis of Manic Episodes. Lithium and Valp are universally recommended by all the guidelines, which is also the recommendation of the unified guideline.

3.1.6. Prophylaxis of Depressive Episodes. Lamotrigine is recommended as the first line by APA and Canadian guidelines while NICE and Maudsley ask it to be used as second line. $\mathrm{Li}$ and Valp have been recommended by all except APA, while Quetiapine has been recommended by both NICE and Maudsley. The unified guideline recommends Lamotrigine followed by Li, Valp, and Quet as drugs to be used in the maintenance of depressive episodes.

3.1.7. Duration of Pharmacotherapy. Only APA and Canadian guidelines have given recommendations on the duration of acute and continuation phase, which, however, vary as seen in the table. The maintenance phase duration, however, is similar across all guidelines and hence the unified guideline recommends treatment in an acute phase up to 12 weeks, in the continuation phase up to 9 months, and in the maintenance phase as 2 years for 1st episode and 5 years to lifetime for subsequent episodes.

3.1.8. Psychosocial Management. The use of psychoeducation has been recommended by only Canadian guidelines with the NICE actively discouraging it. Both NICE and APA as well as recent meta-analysis [14] favor the use of either family focused therapy (FFT) or cognitive behavior therapy (CBT) (Level II evidence), which is the recommendation of the unified guideline also.

\subsection{Management of Depressive Disorder (Table 2)}

3.2.1. Acute Management of Depressive Episode. Since both recent evidence [18-20] and all the guidelines are unanimous in favoring both SSRIs and SNRIs (Serotonin Noradrenaline Reuptake Inhibitors) for the acute management of a depressive episode, this is also the recommendation of the unified guideline.

3.2.2. Management of Atypical Depression. Only the Canadian and NICE guidelines have recommended specific treatments for atypical depression. Recent evidence favors the use of either SSRIs or MAOIs (Monoamine Oxidase Inhibitors) as first line drugs [20], which is also the recommendation of the unified guideline.

3.2.3. Management of Melancholic Depression. Once again, only the Canadian and NICE guidelines have recommended specific treatments for melancholic depression. The unified 


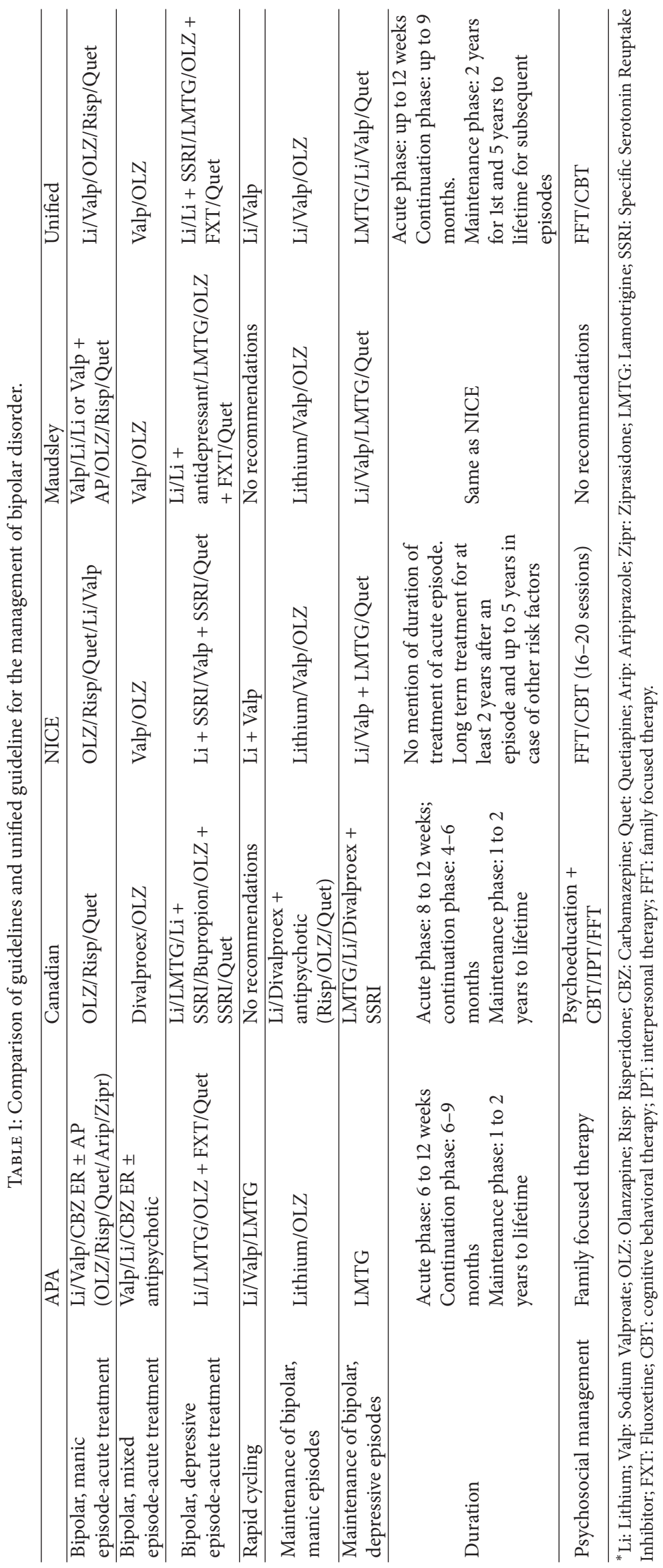




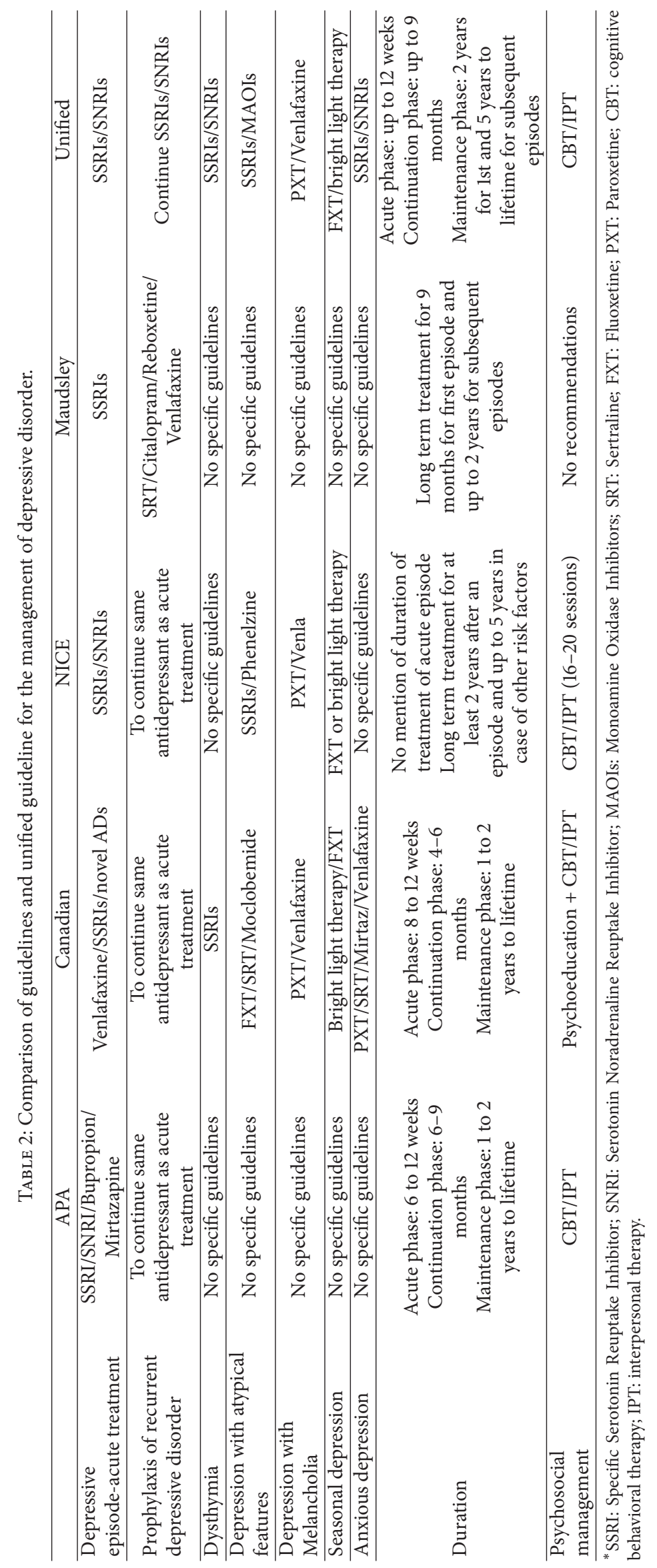


guideline hence recommends the use of either Paroxetine (PXT) or Venlafaxine (Venla) as first line drugs, while recent reviews have also favored tricyclic antidepressants (TCAs) such as amitriptyline and clomipramine which have both noradrenergic and serotonergic activity [20].

3.2.4. Management of Seasonal Depression. Both the Canadian and NICE recommend the use of either bright light therapy or Fluoxetine (FXT), which is also recommended by the unified guideline.

3.2.5. Management of Anxious Depression. No separate recommendations exist for anxious depression by either APA, NICE, or Maudsley. The Canadian guideline recommends the use of either SSRIs like Paroxetine or Sertraline (SRT) or SNRIs like Venlafaxine. This is also supported by the unified guideline.

3.2.6. Management of Dysthymia. Since no separate recommendations exist except by the Canadian guidelines, the unified guideline recommends the use of either SSRIs or SNRIs as first line management.

3.2.7. Prophylaxis of Depressive Disorder. Almost all the guidelines recommend the continuation of the same antidepressant as was initiated for the acute management of the depressive episode. In addition, the Maudsley guideline recommends the use of either Sertraline, Citalopram, Reboxetine, or Venlafaxine, which have been found effective in prophylaxis. The unified guideline recommends the continuation of the same antidepressant, either SSRI or SNRI.

3.2.8. Duration of Pharmacotherapy. There are differing recommendations on the duration of pharmacotherapy, with both APA and Canadian guidelines specifying the duration of acute phase as up to 12 weeks and continuation phase as up to 9 months. In contrast, the Maudsley and NICE guidelines offer no specific recommendation for the acute management. Long term management again differs, with APA and Canadian being similar and NICE and Maudsley differing on the duration. The unified guideline recommends an acute phase treatment of up to 12 weeks, continuation phase up to 9 months, and maintenance phase as 2 years for a first episode and 5 years to lifetime for subsequent episodes.

3.2.9. Psychosocial Management. CBT and interpersonal therapy (IPT) in 16-20 sessions (Level I evidence) are the recommendation of the APA, Canadian, and the NICE guidelines, as well as that of recent reviews [20] and hence that of the unified guideline.

\subsection{Management of Schizophrenia (Table 3)}

3.3.1. Acute Management of Psychotic Episode. With the exception of the Canadian guidelines, all others recommend the use of either SGAs (first line) or FGAs (second line) as standard drugs. The Canadian guideline only recommends the use of SGAs such as Olanz, Risp, or Quetiapine. Based on recent evidence [21], the unified guideline recommends the use of either first or second generation antipsychotics
(SGAs/FGAs) based on clinical and economic needs [22] at a dosage of 300-1000 chlorpromazine (CPZ) equivalents [23].

3.3.2. Prophylaxis of Schizophrenia. All the guidelines and the unified guideline recommend the continued use of the same antipsychotic used to manage the acute episode for prophylaxis [23].

3.3.3. Duration of Pharmacotherapy. The APA and Canadian guidelines recommend similar durations of acute, stabilization, and stable phase treatment. The NICE and Maudsley guidelines recommend acute treatment to last 2 years and give no specific recommendation on duration of prophylaxis. The unified guideline recommends the acute phase treatment to last at least 12 weeks, the stabilization phase to last at least 12 months, and stable phase to last at least 2 years for a first episode and 5 years to lifetime for multiple episodes.

3.3.4. Psychosocial Management. The APA and Canadian guidelines find evidence for and recommend the use of family psychoeducation lasting greater than 9 months, assertive community treatment (ACT), supported employment programs, social skills training (SST), and cognitive behavioral therapy lasting 16-20 sessions. This is also supported by the PORT treatment recommendations [24]. The NICE guideline however finds little or no evidence for psychoeducation and social skills training and hence recommends the use of only 16-session CBT, 10-session family focused therapy, supported employment programs, and Arts therapy. Maudsley guidelines make no specific recommendations. The unified guideline recommendations are therefore the same as the APA guidelines.

\section{Discussion}

Although guidelines have been postulated to improve clinical practice, their implementation has been difficult to achieve due to the characteristics of the guidelines themselves, such as clarity, complexity of treatment recommendations, perceived credibility, use of evidence based medicine, and (pharmaceutical) sponsorship, which have been shown to affect clinicians' acceptance of guidelines [25-28]. To improve acceptance, one would need to formulate an ideal guideline that would be derived from a comprehensive literature review and would explicitly assess the quality of supporting research studies and the methods used for synthesizing evidence. Such a guideline would make recommendations not only for the pharmacological management but also for assessment and psychosocial interventions during both the acute and the maintenance phases of the illness.

Earlier studies have attempted to compare the applicability and evidence strength [29] or the methodological and scientific quality [30] of different guidelines, which have mainly involved schizophrenia. This paper comprehensively looks at and compares the content quality of the four mostfollowed guidelines for the three most serious mental disorders, namely, schizophrenia, bipolar disorder, and recurrent depressive disorder. It evaluates these guidelines on the presence of evidence based medicine and then makes a 


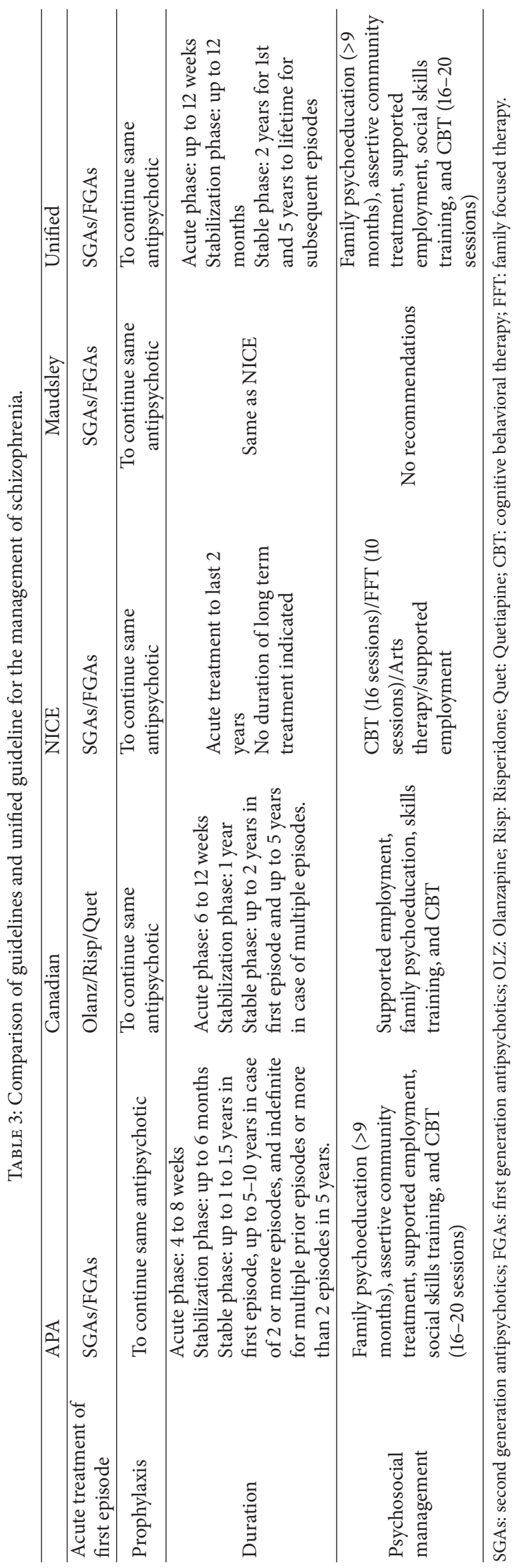


unified guideline recommendation, which may be useful to clinicians and students alike. It also observes that most guidelines gave more detailed recommendations in the field of pharmacotherapy while shedding little light on psychosocial management. In contrast to the advice on psychotropic medication, recommendations for psychosocial treatment were very general and nonspecific in many cases. The choice of psychotropic medications was also a major concern. Whereas in some fields recommendations were quite similar among guidelines (e.g., Valproate in case of acute management of mixed episodes, antidepressant use, and duration of long term antipsychotic treatment), others differed widely (recommendations on psychosocial management and duration of acute treatment of mood episode). Such differences actually make it difficult for clinicians to evaluate and implement differing recommendations in their daily practice. The unified guideline attempts to bridge this gap, by evaluation of both the guidelines and available evidence, to make a clear recommendation. The authors hope that this would go a long way in helping clear some of the confusion that has crept in due to the use of different guidelines across the world.

In addition, the authors feel that an internationally acceptable and culturally fair set of recommendations could be developed and form the framework for further elaboration on a national or local basis. This could be facilitated by independent and international organizations such as the WHO and the WPA, after which these could then be used for adaptation to different cultural, economic, and other backgrounds in collaboration with individual stakeholders of the respective countries and regions.

\section{Clinical Recommendations}

(i) Different guidelines suggest different management strategies for bipolar disorder, major depressive disorder, and schizophrenia.

(ii) A unified guideline, formulated taking into account recent meta-analytical evidence, may help clear the confusion brought about by these differing guidelines.

(iii) International organizations such as the WPA or WHO may help formulate a unified guideline, which may then be modified to meet national or local needs.

\section{Conflict of Interests}

The authors declare that there is no conflict of interests.

\section{References}

[1] S. H. Woolf, "Practice guidelines, a new reality in medicine: II. Methods of developing guidelines," Archives of Internal Medicine, vol. 152, no. 5, pp. 946-952, 1992.

[2] R. Jackson and G. Feder, "Guidelines for clinical guidelines," British Medical Journal, vol. 317, no. 7156, pp. 427-428, 1998.

[3] P. Littlejohns, F. Cluzeau, R. Bale, J. Grimshaw, G. Feder, and S. Moran, "The quantity and quality of clinical practice guidelines for the management of depression in primary care in the UK," British Journal of General Practice, vol. 49, no. 440, pp. 205-210, 1999.
[4] T. M. Shaneyfelt, M. F. Mayo-Smith, and J. Rothwangl, "Are guidelines following guidelines?. The methodological quality of clinical practice guidelines in the peer-reviewed medical literature," Journal of the American Medical Association, vol. 281, no. 20, pp. 1900-1905, 1999.

[5] M. D. Cabana, C. S. Rand, N. R. Powe et al., "Why don't physicians follow clinical practice guidelines?: a framework for improvement," Journal of the American Medical Association, vol. 282, no. 15, pp. 1458-1465, 1999.

[6] Practice guideline 2006: American Psychiatric Association, http://www.psych.org/mainmenu/psychiatricpractice/practiceguidelines_l.aspx.

[7] Canadian Psychiatric Association, "Clinical Practice Guidelines-for the treatment of depressive disorders," Canadian Journal of Psychiatry, vol. 46, no. S1, pp. 1S-90S, 2001.

[8] Canadian Psychiatric Association, "Clinical Practice Guidelines-treatment of schizophrenia," Canadian Journal of Psychiatry, vol. 50, no. S1, pp. 1S-56S, 2005.

[9] L. N. Yatham, S. H. Kennedy, C. O’Donovan et al., "Canadian Network for Mood and Anxiety Treatments (CANMAT) guidelines for the management of patienst with bipolar disorder: consensus and controversies," Bipolar Disorders, Supplement, vol. 7, no. 3, pp. 5-69, 2005.

[10] L. N. Yatham, S. H. Kennedy, C. O'donovan et al., "Canadian Network for Mood and Anxiety Treatments (CANMAT) guidelines for the management of patients with bipolar disorder: update 2007," Bipolar Disorders, vol. 8, no. 6, pp. 721-739, 2006.

[11] National Institute for Health \& Clinical Excellence, "NICE guidelines for Mental Health \& Behavioral conditions," 2010, http://guidance.nice.org.uk/Topic/MentalHealthBehavioural.

[12] D. Taylor, C. Paton, and S. Kapur, The Maudsley Prescribing Guidelines, Informa Healthcare, London, UK, 10th edition, 2009.

[13] A. Cipriani, C. Barbui, G. Salanti et al., "Comparative efficacy and acceptability of antimanic drugs in acute mania: a multipletreatments meta-analysis," The Lancet, vol. 378, no. 9799, pp. 1306-1315, 2011.

[14] G. S. Malhi, D. Adams, L. Lampe et al., "Clinical practice recommendations for bipolar disorder," Acta Psychiatrica Scandinavica, vol. 119, no. s439, pp. 27-46, 2009.

[15] M. A. Frye, "Bipolar disorder-a focus on depression," The New England Journal of Medicine, vol. 364, no. 1, pp. 51-59, 2011.

[16] A. M. A. Nivoli, F. Colom, A. Murru et al., "New treatment guidelines for acute bipolar depression: a systematic review," Journal of Affective Disorders, vol. 129, no. 1-3, pp. 14-26, 2011.

[17] M. L. Zupancic, "Role of atypical antipsychotics in rapid cycling bipolar disorder: a review of the literature," Annals of Clinical Psychiatry, vol. 23, no. 2, pp. 141-149, 2011.

[18] J. R. T. Davidson, "Major depressive disorder treatment guidelines in America and Europe," The Journal of Clinical Psychiatry, vol. 71, no. E1, p. e04, 2010.

[19] D. J. Nutt, J. R. T. Davidson, A. J. Gelenberg et al., "International consensus statement on major depressive disorder," The Journal of Clinical Psychiatry, vol. 71, no. E1, p. e08, 2010.

[20] G. S. Malhi, D. Adams, R. Porter et al., "Clinical practice recommendations for depression," Acta Psychiatrica Scandinavica, vol. 119, no. 439, pp. 8-26, 2009.

[21] J. M. Kane and C. U. Correll, "Pharmacologic treatment of schizophrenia," Dialogues in Clinical Neuroscience, vol. 12, no. 3, pp. 345-357, 2010. 
[22] R. Tandon, R. H. Belmaker, W. F. Gattaz et al., "World Psychiatric Association Pharmacopsychiatry Section statement on comparative effectiveness of antipsychotics in the treatment of schizophrenia," Schizophrenia Research, vol. 100, no. 1-3, pp. 2038, 2008.

[23] J. Kreyenbuhl, R. W. Buchanan, F. B. Dickerson, and L. B. Dixon, "The schizophrenia patient outcomes research team (PORT): updated treatment recommendations 2009," Schizophrenia Bulletin, vol. 36, no. 1, pp. 94-103, 2010.

[24] L. B. Dixon, F. Dickerson, A. S. Bellack et al., "The 2009 schizophrenia PORT psychosocial treatment recommendations and summary statements," Schizophrenia Bulletin, vol. 36, no. 1, pp. 48-70, 2010.

[25] D. A. Davis and A. Taylor-Vaisey, "Translating guidelines into practice. A systematic review of theoretic concepts, practical experience and research evidence in the adoption of clinical practice guidelines," Canadian Medical Association Journal, vol. 157, no. 4, pp. 408-416, 1997.

[26] R. S. A. Hayward, "Clinical practice guidelines on trial," Canadian Medical Association Journal, vol. 156, no. 12, pp. 1725-1727, 1997.

[27] L. I. Solberg, M. L. Brekke, C. J. Fazio et al., "Lessons from experienced guideline implementers: attend to many factors and use multiple strategies," The Joint Commission Journal on Quality Improvement, vol. 26, no. 4, pp. 171-188, 2000.

[28] L. Samalin, S. Guillaume, C. Auclair, and P.-M. Llorca, "Adherence to guidelines by french psychiatrists in their real world of clinical practice," Journal of Nervous and Mental Disease, vol. 199, no. 4, pp. 239-243, 2011.

[29] K. K. Milner and M. Valenstein, "A comparison of guidelines for the treatment of schizophrenia," Psychiatric Services, vol. 53, no. 7, pp. 888-890, 2002.

[30] W. Gaebel, S. Weinmann, N. Sartorius, W. Rutz, and J. S. McIntyre, "Schizophrenia practice guidelines: international survey and comparison," British Journal of Psychiatry, vol. 187, pp. 248255, 2005. 


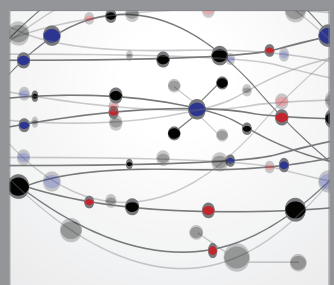

The Scientific World Journal
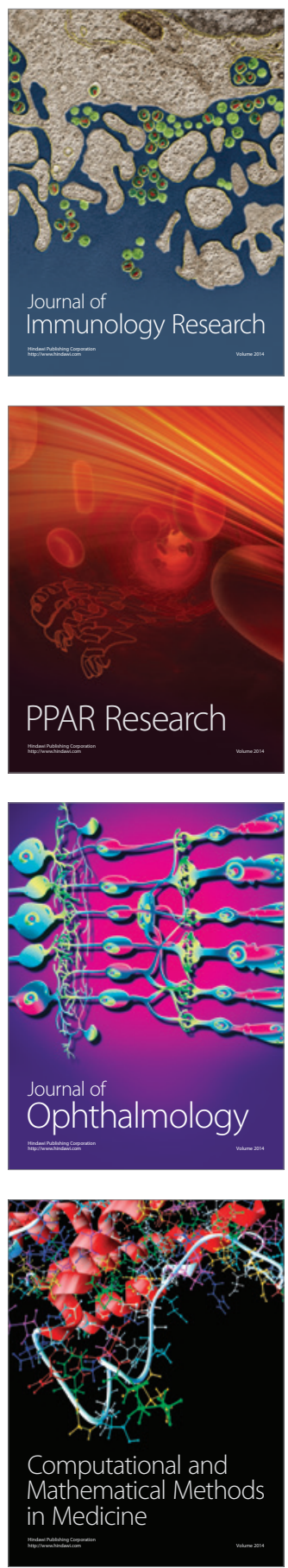

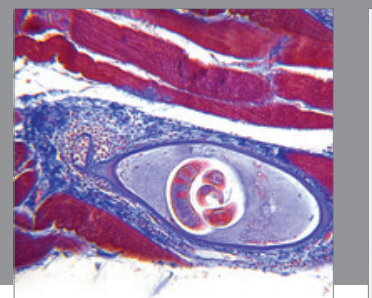

Gastroenterology

Research and Practice
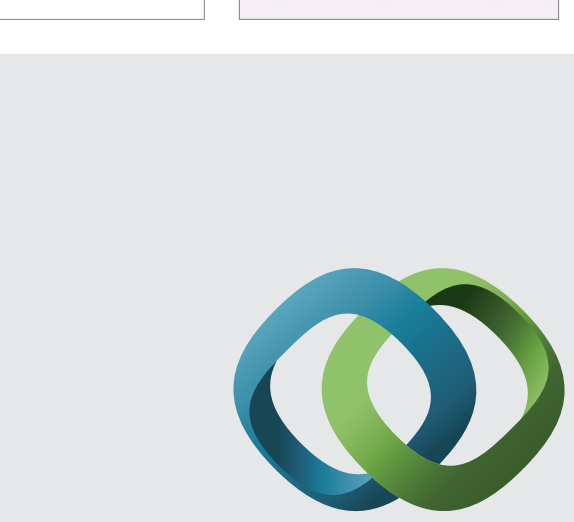

\section{Hindawi}

Submit your manuscripts at

http://www.hindawi.com
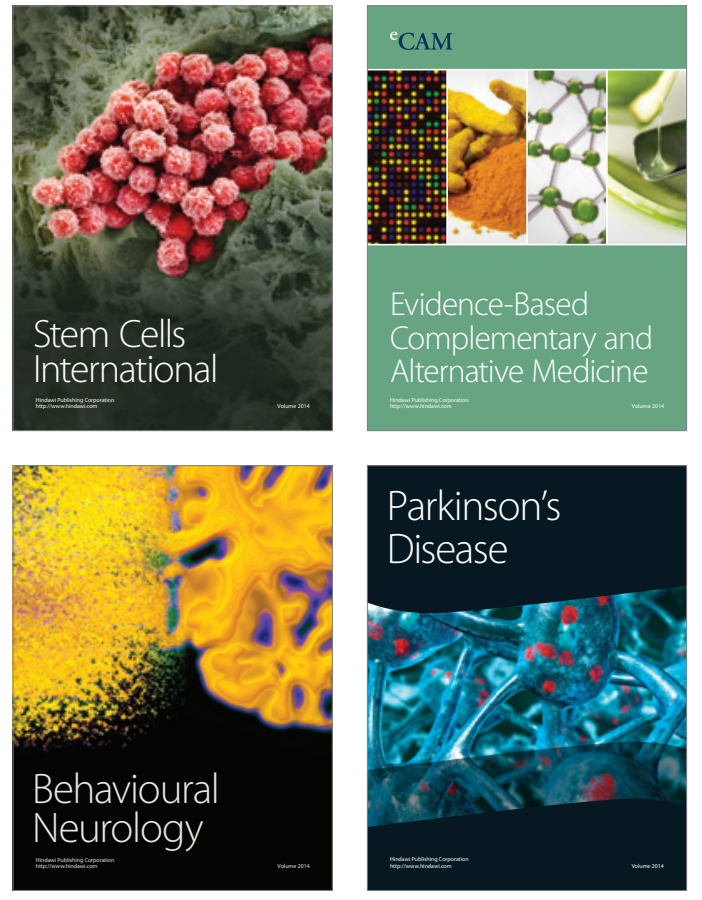
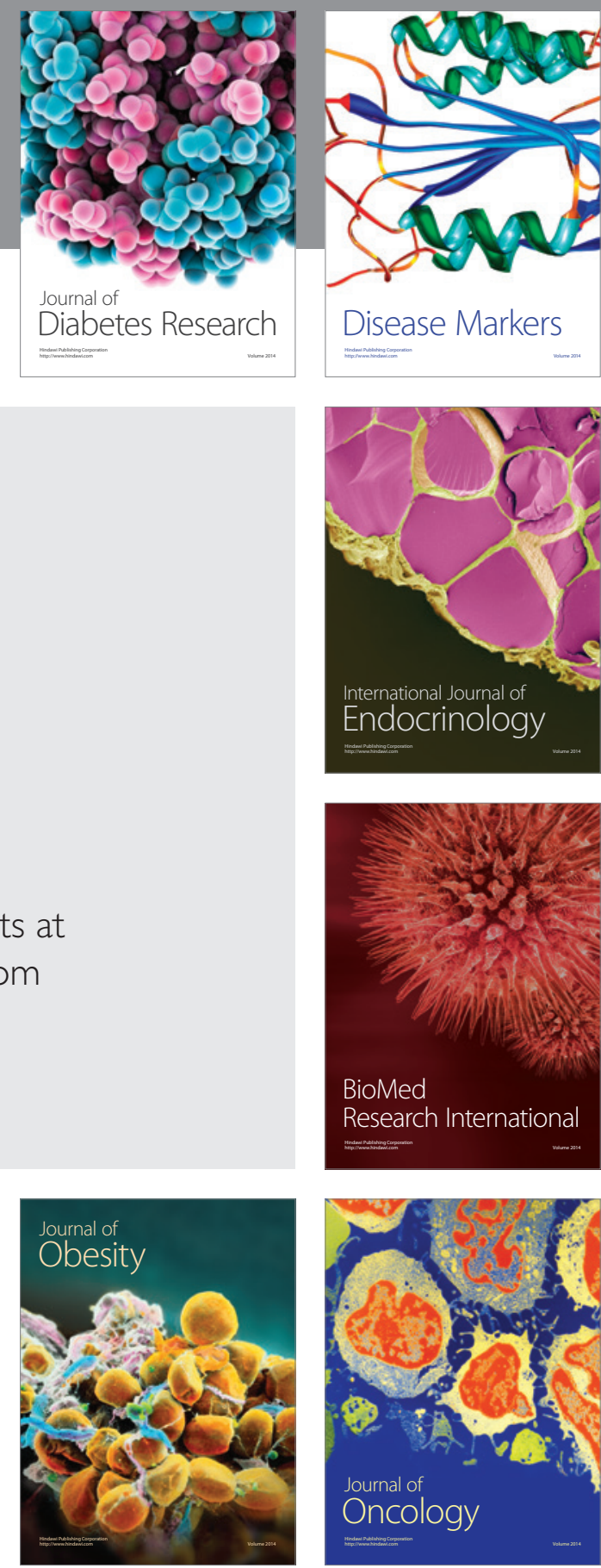

Disease Markers
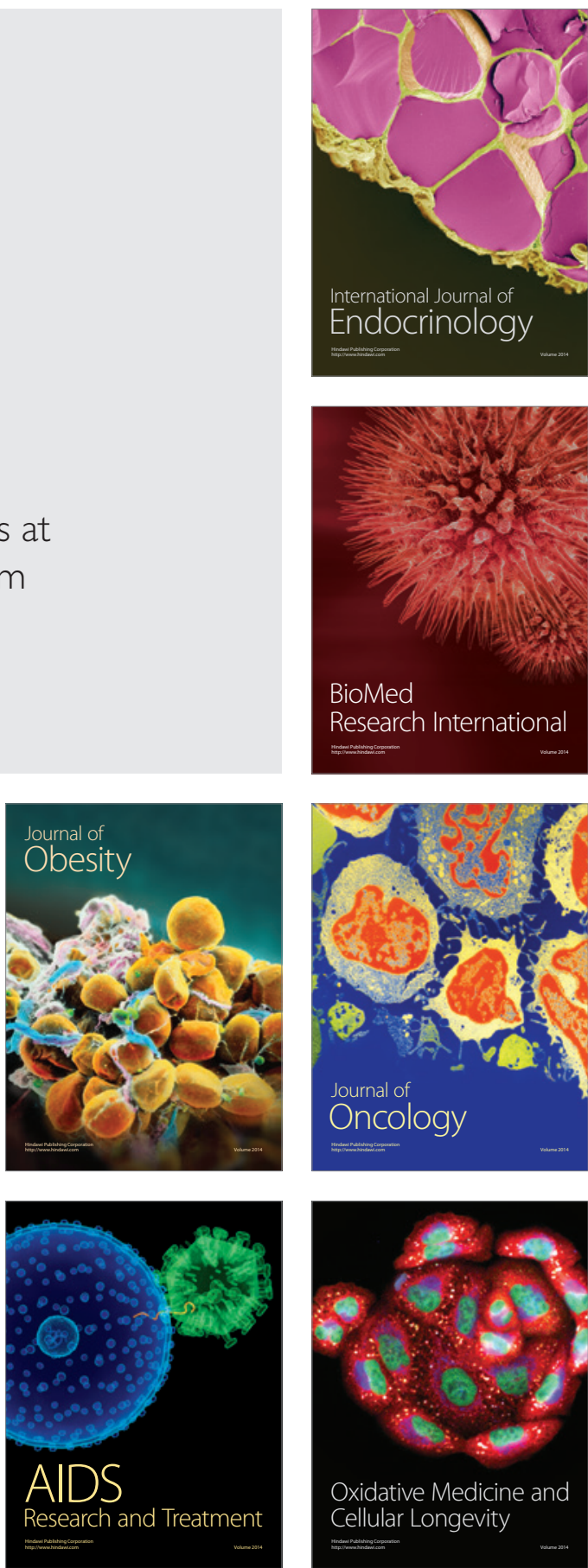